\title{
Prosaccadic and antisaccadic performance of the athletes in different types of sports.
}

\author{
Alpaslan Yilmaz ${ }^{1,2 *}$, Metin Polat ${ }^{1}$ \\ ${ }^{1}$ Department of Sport and Health, Physical Education and Sport College, Erciyes University, Kayseri, Turkey \\ ${ }^{2}$ Department of Psychiatry, SoCAT Lab, Ege University School of Medicine, Bornova, Izmir, Turkey
}

\begin{abstract}
The perception of the visual environment plays the most prominent role for athletes of some sports types. Saccades are the fastest movements of eye balls to launch the image of any peripheral visual object to fovea. Some researchers previously reported that athletes have better saccadic performance than non-athletes. We aimed to investigate prosaccadic and antisaccadic performances of athletes from various sports, which have different visual field changing velocities. Totally 40 male athletes were divided into four groups (tennis players, volleyball players, basketball players, swimmers), and each of them included 10 athletes. 10 non-athletes were formed as the control group. Our results indicated that tennis players and volleyball players had faster prosaccadic velocity than basketball players, swimmers and non-athletes groups. We found shorter antisaccadic latency in tennis, volleyball and basketball players than non-athletes. Swimmers' antisaccadic latency values were significantly different from neither basketball players nor non-athletes. We considered from these findings that gaining experience in some branches may improve planning of voluntary saccades and prosaccadic velocity. These improvements are evident in fast ball game players such as tennis and volleyball.
\end{abstract}

Keywords: Prosaccade, Antisaccade, Sport.

Accepted on November 9, 2017

\section{Introduction}

In many types of sports, athletes are forced to recognize fastchanging visual field. Especially in fast ball games, the success of an athlete depends strongly on the power of determining the location of the ball and other players as quickly as possible. In some cases, athletes have to make a saccadic eye movement to the direction of a visual stimulus, yet sometime they have to move their eyes to another direction of visual stimulus. A visual object can be grabbed quicker by shortening saccade latency or increasing saccade velocity. A prosaccade is a saccadic eye movement towards a visual stimulus [1,2] and prosaccadic eye movements in athletes are reported to be faster than non-athletes [3-6]. A saccade to the opposite direction of visual stimulus is an antisaccade $[1,2]$ and athletes are reported to have shorter latency than non-athletes [7]. These are not only advantages of the visual field scanning of athletes. Zwierko reported that handball players have better peripheral vision than non-players [8], and Piras et al. reported that experienced volleyball players use different scan strategies from novice ones [9]. In other words, despite the presence of lots of visual stimuli in their visual fields, superior athletes can achieve saccade to the right target at the right time. In order to achieve this, athletes must have strong suppression of prosaccades and faster preparation of voluntary [10]. Fischer and Weber reported that training leads to a shorter production phase of antisaccade [11]. On the other hand, Abernethy and Wood argue that visual training cannot provide improvements in either basic visual function or motor performance [12]. But some recent studies showed that visual training brings about enhancement of visual functions including saccadic eye movements [13].

Each sport has its own visual scene. In some sports, athletes face extremely fast changing visual scenes but in some sports they do not. For example, tennis players need faster scan of the visual environment [13] than other athletes such as swimmers. When the ball is moving fast, athletes have to make saccades to capture the image of the ball. Tracing an object moving with a velocity under $100 \mathrm{degree} / \mathrm{sec}$ is adequate to pursuit eye movement system [14]. In this situation, athletes need less voluntary saccades. It can be suggested that, the characteristics of any kind of sports may improve different eye movement control mechanisms of the athletes. In this study, we intend to find out prosaccadic and antisaccadic performances of athletes from various sports, which have different visual field changing velocities. The aim of the present research was to assess how tennis (where ball moves remarkably fast), volleyball (where ball moves relatively slower, but players should visually track of the opponent players), basketball (where a bigger ball is used, but the game is played more slowly, yet visual information is also beneficial) and swimming (where it is not 
critically needed to scan the visual scene) affected prosaccadic and antisaccadic performance.

\section{Materials and Methods}

\section{Participants}

Totally 40 male athletes were divided into four groups (Tennis players' age, $\mathrm{M}=21.9 \pm 1.4$; volleyball players' age, $\mathrm{M}=21.6 \pm$ 1.6; basketball players' age, $\mathrm{M}=22.1 \pm 1.5$; swimmers' age, $\mathrm{M}=21.8 \pm 1.2$ ) and each of them included 10 athletes. 10 nonathletes $(M=21.6 \pm 1.4)$ were prepared as the control group. In this research, all participants did not have any sight problem and drug-free for the last $48 \mathrm{~h}$. Athletes who hadn't made regular exercises at least four years in their own branches excluded from the study. All of the participants accepted voluntarily to take part in this test. Test did not include any invasive or hazardous procedure.

\section{Procedure}

The subjects sat in a dark, quiet room, and their heads stabilized in a headrest. Horizontal eye movements were recorded binocularly with using electrooculography (Biopack MP-30, filter: $0.05-35 \mathrm{~Hz}$ ) at a $1000 \mathrm{~Hz}$ sampling rate. Surface electrodes were placed $1 \mathrm{~cm}$ laterally to the outer canthi with a reference electrode at the center of the forehead, after skin scarified.

Each subject faced in front of the panel at a distance of $200 \mathrm{~cm}$ away which included three visual Light-Emitting Diode (LED) targets ( $0.11 \mathrm{o}$ in diameter): one of LED target was fixed at midline of the panel for the initial condition at zero degrees, and two of them were fixed at $15^{\circ}$ to the left and the right side of central point. Before the test, the subjects stayed in a dark condition in the room for adaptation for about ten minutes.

At the beginning of each test, participants were asked to look at the LED in the center. During the test, in an arbitrary manner and time interval (min: $1 \mathrm{~s}$, max: $5 \mathrm{~s}$ ), one of the left or right target LEDs lightened for one second and after that the LED on the central point lightened again. In the prosaccade task, participants were asked to look at the LED lightened. In the antisaccade task, they were asked to look at the opposite direction of the lightened LED, after that, they were asked to look at the LED on the central location when lightened. For each direction, the procedure was repeated ten times to produce quite twenty pro and antisaccade of each subject. All stimulus patterns were used according to gap paradigm.

Any anticipatory and reflexive saccade which did have latency shorter than $100 \mathrm{~ms}$ was not accepted for consideration. Looking at the target LED during antisaccade test was considered as antisaccade error. As a result of the elimination of any saccade record (due to blink artifact, antisaccade error, reflexive saccade, suspicious amplitude etc.) was made immediate after the recording, at least twenty clean pro and anti-saccades were recorded for each participant.

Statistical analyses were made by using one-way ANOVA test. Post-hoc analysis using Tukey procedure was performed to examine the different suggested by ANOVA.

\section{Results}

Latency and velocity mean \pm SEM values of pro and antisaccade of five groups are illustrated in Table 1. According to ANOVA results, there were significant differences among groups in antisaccade latency $\left(\mathrm{f}_{4,45}=8.88 ; \mathrm{P}<0.05\right)$ and prosaccade velocity $\left(\mathrm{f}_{4,45}=10.74 ; \mathrm{P}<0.05\right)$.

In light of these results, there was no significant difference, in the comparison of antisaccade velocity or prosaccade latency, within groups by ANOVA.

Post-hoc analysis of prosaccade velocity showed that tennis players were significantly different from basketball players, swimmers and non-athletes. There was no significant difference between tennis players and volleyball players in prosaccade velocity. Prosaccade velocity of basketball players, volleyball players, and swimmers was also not significantly different (Table 2).

Post-hoc analysis of antisaccade latency showed that tennis players were significantly different from swimmers and nonathletes. There was no significant difference between basketball players and swimmers in antisaccade latency. Antisaccade latency of non-athletes and swimmers were also not significantly different (Table 3). Prosaccade and antisaccade velocity and latency values of all groups are shown in Figures 1 and 2 respectively.

Table 1. Mean \pm SE values of prosaccadic and antisaccadic latency and velocity of all groups. For each row, any values having the same letter are not significantly different.

\begin{tabular}{|c|c|c|c|c|c|c|}
\hline & TP & VP & BP & sw & NA & $F_{4,45}$ \\
\hline Prosaccade latency (ms) & $199.99 \pm 4.59$ & $203.64 \pm 5.23$ & $205.22 \pm 5.80$ & $216.40 \pm 6.10$ & $212.83 \pm 6.95$ & 1.37 \\
\hline Prosaccade velocity $(\% / s)$ & $255.11 \pm 8.31^{a}$ & $242.15 \pm 7.22^{a b}$ & $222.29 \pm 5.77^{\mathrm{bc}}$ & $217.56 \pm 4.93^{c}$ & $196.29 \pm 7.91^{c}$ & 10.74 \\
\hline Antisaccade latency (ms) & $238.62 \pm 12.67^{a}$ & $240.51 \pm 10.37^{a}$ & $266.08 \pm 9.14^{\mathrm{ab}}$ & $289.07 \pm 8.86^{b c}$ & $307.21 \pm 8.78^{c}$ & 8.88 \\
\hline Antisaccade velocity $\left(d^{\circ} / s\right)$ & $184.54 \pm 8.38$ & $174.65 \pm 6.08$ & $194.78 \pm 10.34$ & $180.57 \pm 6.67$ & $167.93 \pm 6.17$ & 1.68 \\
\hline
\end{tabular}

TP: Tennis Players; VP: Volleyball Players; BP: Basketball Players; SW: Swimmers; NA: Non-Athletes. 


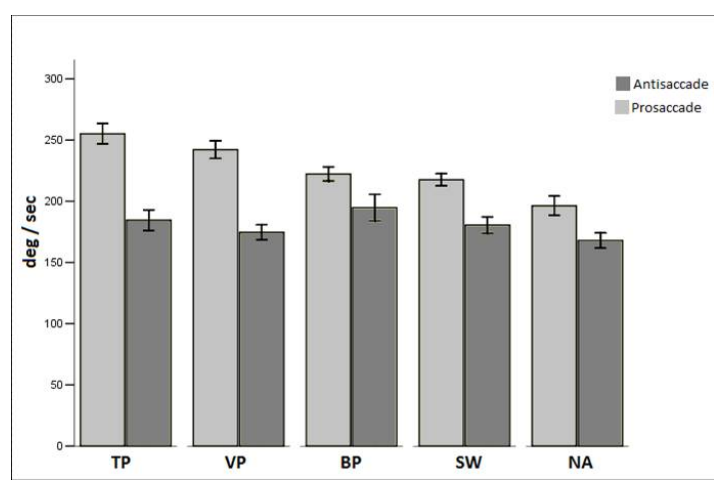

Figure 1. Prosaccade and antisaccade velocity of study groups. Bars represent mean \pm SE. (TP: Tennis Players; VP: Volleyball Players; BP: Basketball Players; SW; Swimmers; NA: Non-Athletes).

Table 2. Comparison of prosaccade velocity in different groups.

\begin{tabular}{ll}
\hline Prosaccade velocity & Significant difference \\
\hline TP-VP & $P>0.05$ \\
\hline TP-BP & $P<0.05$ \\
\hline TP-SW & $P<0.01$ \\
\hline TP-NA & $P<0.01$ \\
\hline VP-BP & $P>0.05$ \\
\hline VP-SW & $P>0.05$ \\
\hline VP-NA & $P<0.01$ \\
\hline BP-SW & $P>0.05$ \\
\hline BP-NA & $P>0.05$ \\
\hline SW-NA & $P>0.05$ \\
\hline
\end{tabular}

Table 3. Comparison of antisaccade latency in different groups.

\begin{tabular}{ll}
\hline Antisaccade latency & Significant difference \\
\hline TP-VP & $P>0.05$ \\
\hline TP-BP & $P>0.05$ \\
\hline TP-SW & $P<0.01$ \\
\hline TP-NA & $P<0.01$ \\
\hline VP-BP & $P>0.05$ \\
\hline VP-SW & $P<0.05$ \\
\hline VP-NA & $P<0.01$ \\
\hline BP-SW & $P>0.05$ \\
\hline BP-NA & $P<0.05$ \\
\hline SW-NA & $P>0.05$ \\
\hline
\end{tabular}

TP: Tennis Players; VP: Volleyball Players; BP: Basketball Players; SW: Swimmers; NA: Non-Athletes.

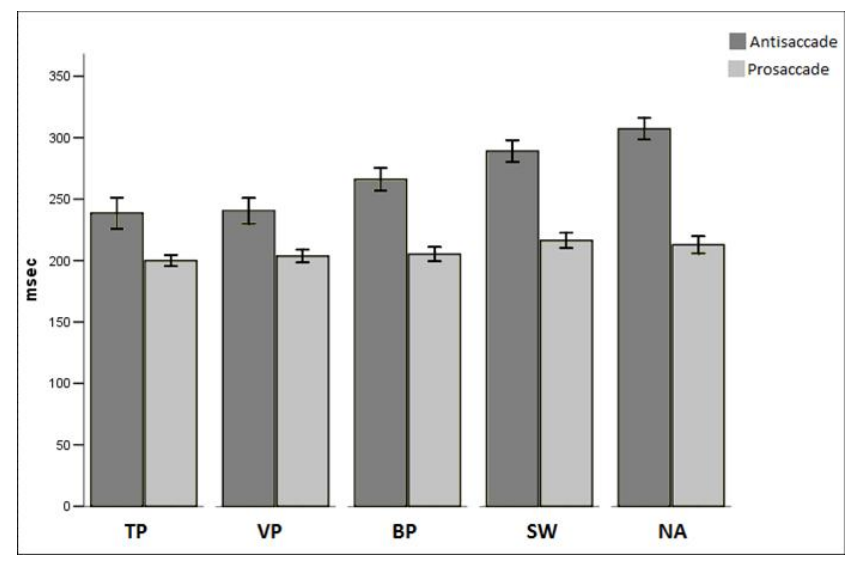

Figure 2. Prosaccade and antisaccade latency time of study groups. Bars represent mean \pm SE. (TP: Tennis Players; VP: Volleyball Players; BP: Basketball Players; SW: Swimmers; NA: Non-Athletes).

\section{Discussion}

According to findings of the research, we could not find any difference in prosaccadic latency time among the research groups. This conclusion is supported and consistent with the evidence reported by Lenoir et al. [4], Fujiyawa et al. [15] and Babu et al. [16]. In a similar research, only Morrillo et al. [17] reported that professional shooters have shorter prosaccadic latency than the control group.

We found significant differences in prosaccadic velocity between athletes and non-athletes according to some of past and similar researches in the past [3-6]. We were unable to find any research that compared types of sports in terms of prosaccadic velocity in the literature. There was no significant difference between tennis and volleyball players but both of these participants were significantly different prosaccadic velocity from non-athletes. In the other exam, tennis and volleyball players should follow the opponents and balls remarkably quickly during the competition. We thought that volleyball and tennis branches had similar visual scenes such as ball speed, ball control and body movement in nature. Interestingly, there was no significant difference between volleyball and basketball players in prosaccadic velocity. However, the basketball players showed no significant difference in terms of prosaccadic velocity compared to both swimmers and non-athletes. In this study, we can see that gaining experience in basketball may increase prosaccadic velocity as much as volleyball training, but on the other hand, they may not improve and affect prosaccadic velocity compared to tennis training. When we compared the prosaccadic velocity of swimmers to non-athletes, the basketball and volleyball players, we found no significant differences. We thought that the athletes' eye movement toward a visual object did not play a key role in swimming; we expected swimmers to display similar prosaccadic velocity to non-athletes. On the other hand, swimmers' prosaccadic velocities were not different from volleyball players' too. 
According to this situation, it could be said that swimmers do not need to improve prosaccadic velocity for their performance, but as an athlete, they can get an opportunity to improve the prosaccadic velocity, too. Jafarzadehpur et al. [6] reported that athletes have better prosaccadic performance based on their experience levels in volleyball than non-athletes. Because Jafarzadehpur et al. [6] evaluated prosaccadic performance in saccade count per a constant time, it was not clear whether prosaccadic latency or velocity was affected.

In our research, the findings showed that there was no antisaccadic velocity difference in the control and research groups. But we found significant differences in antisaccadic latency among the three (tennis players, volleyball players, basketball players) athlete groups compared to the non-athlete group. Our results showed that no significant difference was present between swimmers and non-athletes. It was expected that swimming did not have a fast-changing visual field as a training environment. Yet, our results indicated that swimmers' antisaccadic latency was not different from basketball players and non-athletes although basketball players had different antisaccadic latency from non-athletes. Shorter antisaccadic latency in athletes compared to non-athletes was a common finding in the literature $[3,17]$. Some studies comparing novice and experienced athletes in antisaccadic latency reported shorter antisaccadic latency among expert players than novice ones $[4,7,15]$. Fischer and Weber [11] reported that training, which included antisaccade task, provided shorter antisaccade latency. Some athletic trainings and competitions may force athletes to make antisaccade. Anyone who intends to make an antisaccade must first suppress the automatic response to look at the object and then convert the position of the stimulus into a voluntary motor command to look away from the target [10]. Due to the capability of suppression of reflexive eye movement, athletes are more advantageous to move their eyes towards where they want. We thought that training and having higher visual attention level may play a decisive function to be able to suppress reflexive prosaccades in athletes. For this reason experienced athletes show more effective gaze strategies than novice athletes [9].

Hence, in this paper we assume that different types of sports may improve saccadic system consistent with their visual conditions. For example, both tennis and volleyball had a fast changing visual scene, and both tennis and volleyball players had better prosaccadic and antisaccadic performance than swimmers and partly basketball players.

In conclusion, we suggest that some types of sports-especially critical need identification of the visual environment as quickly as possible-enhance saccadic system naturally. On this basis, we have the same opinion with Abernethy and Wood [12] that extra visual training is not necessary to enhance athletic performance.

\section{References}

1. Rafal R, Egly R, Rhodes D. Effects of inhibition of return on voluntary and visually guided saccades. Can J Exp Psychol 1994; 48: 284-300.

2. Goldring J, Fischer B. Reaction times of vertical prosaccades and antisaccades in gap and overlap tasks. Exp Brain Res 1997; 113: 88-103.

3. Di Russo F, Pitzalis S, Spinelli D. Fixation stability and saccadic latency in élite shooters. Vision Res 2003; 43: 1837-1845.

4. Lenoir M, Crevits L, Goethals M, Wildenbeest J, Musch E. Are better eye movements an advantage in ball games? A study of prosaccadic and antisaccadic eye movements. Percept Mot Skills 2000; 91:546-52.

5. Lenoir M, Crevits L, Goethals M, Duyck P, Wildenbeest J, Musch E. Saccadic eye movements and finger reaction times of table tennis players of different levels. Neuroophthalmol 2000; 24: 335-338.

6. Jafarzadehpur E, Aazami N, Bolouri B. Comparison of saccadic eye movements and facility of ocular accommodation in female volleyball players and nonplayers. Scand J Med Sci Sports 2007; 17: 186-190.

7. Crevits L, Goethals M, Lenoir M, Wildenbeest J, Musch E. Voluntary saccades in fast ball games. Neuroophthalmol 2000; 24: 331-334.

8. Zwierko T. Differences in peripheral perception between athletes and nonathletes. J Human Kinetics 2007; 19: 53-62.

9. Piras A, Lobietti R, Squatrito S. A study of saccadic eye movement dynamics in volleyball: comparison between athletes and non-athletes. J Sports Med Phys Fitness 2010; 50: 99-108.

10. Munoz DP, Everling S. Look away: the anti-saccade task and the voluntary control of eye movement. Nat Rev Neurosci 2004; 5: 218-228.

11. Fischer B, Weber H. Characteristics of anti saccades in man. Exp Brain Res 1992; 89: 415-424.

12. Abernethy B, Wood JM. Do generalized visual training programmes for sport really work? An experimental investigation. J Sports Sci 2001; 19: 203-222.

13. Maman P, Gaurang S, Shadu JS. The effect of vision training on performance in tennis players. Serb J Sports Sci 2011; 5: 11-16.

14. Spering M, Gegenfurtner KR. Contextual effects on motion perception and smooth pursuit eye movements. Brain Res 2008; 1225: 76-85.

15. Fujiwara K, Kiyota N, Maekawa M, Kunita K, Kiyota T, Maeda K. Saccades and prefrontal hemodynamics in basketball players. Int J Sports Med 2009; 30: 647-651.

16. Babu RJ, Lillakas L, Irving EL. Dynamics of saccadic adaptation: differences between athletes and nonathletes. Optom Vis Sci 2005; 82: 1060-1065.

17. Morrillo M, Di Russo F, Pitzalis S, Spinelli D. Latency of prosaccades and antisaccades in professional shooters. Med Sci Sports Exerc 2006; 38: 388-394. 
Prosaccadic and antisaccadic performance of the athletes in different types of sports

\section{*Correspondence to}

Alpaslan Yilmaz

Department of Sport and Health
Physical Education and Sport College

Erciyes University

Turkey 